been brought into apposition; as also cicatrices, or spots on the cornea, must not be mistaken for cataract opacities. It is enough to direct attention to this point to prevent a mistake.

When, however, on the other hand, the transparency of the lens is affected by general invasion of opacity, the ophthalmoscope is not so available. I am speaking of the very commencement of the affection, when as yet the ere begins to fail a little, especially in viewing distant objects; for somewhat later there are almost always distinct, although irregular markings of more opaque parts, but then the cataract must be so far advanced is to be readily visible; the degree of light then which is necessary to illuminate the eye that the interior may be seen, may render the slight haze in the lens imperceptible. I have proved this over and over. Of course, the presence of cataract in any degree must intercept light; but as the power of individual eyes to receive illumination varies, it is hardly possible to tell whether the greater or lesser degree of this is due to the one or the other cause. At first, I thought that so long as the fundus of the eye was visible, and the optic disc conld be seen well defined, the lens could not be cataractous: now I know the fallacy of such a test, and am well aware that, with the lamp-flame sufficiently intense, it would require cataract far beyond the stage of incipiency to shut out the light.

Should the one eye only be concerned, the difficulty may often be overcome by a comparative exarnination of the other. Where both are involved there must often be doubt, which increases with the age of the patient, because the blackness of the pupil decreases after adolescence, and rapidly after manhood.

I pass now to that part of the subject in which the loss of blackness of the pupil in elderly persons, consequent on the amber change in the lens, is sometimes supposed to be cataract. This was a source of frequent mistakes up to a few years ago; very often there was no means of avoiding it. Here the negative evidence afforded by the ophthalmoscope in the absence of strix, or opaque spots, leaves notling to be desired in the very large majority of cases. Of course, there apply here the same remarks just made about the difficulty of detecting incipient uniform opacity of the lens, with the addition of somewhat greater force, since the eye of a soung person is more easily illuminated than that of an old one.

I may say, however, that when proper care and attention are besiowed, it can be but seldom that errors will be made, except both eyes are cataractous. But a wrong diagnosis is just possible. It has occurred to me lately to know that several London surgeons, all in extensive ophthalmic practice, and any one of whom would be considered zuthority in such a matter by the profession, have been divided, in a particular case, as to the existence or non-existence of cataract in both eyes, after a most prinstaking examination of an elderly lady.

I have yet to speak of one important pathological change in the eye, which concerns us much in the diagnosis of cataract-I mean haziness, or opacity of the vitreous humour. I suspect it must seem as though I have ignored the suljective symptoms of cataract, of which so much has been written in former years, as well as the careful distinctions made between them and amaurosis and glaucoma. The fact is, that they are worth nothing; so little indeed, as to be in themselves useless in a diagnostic point of view, and this chiefly because they are closely imitated by disease of another part of the eye, the vitreous humour, of which till now we were wholly ignorant. No one ever wrote or taught anything about haziness or mistiness of the vitreous humour, becanse no such state was known to exist. To the presence of such an abnormal condition must be referred many blunders and supposed anomalous states, and, I may add, many errors as to the suspected pre. sence of cataract. Only a fortnight ago I was consulted by a nobleman holding a conspicuous place in the political world, who thought that he had cataract, having been told by a London surgeon that he was so afflicted. The answers to my questions were just what I should expect from an intelligent patient who had a lens slightly opaque. I thought there was cataract, although I could not at a glance perceive any indication of it in the pupil ; but as a matter of caution I reserved my opinion till I examined the eye with the ophthalmoscope, when I satisfied myself that the lens was clear, but the vitreous humour was diseased.

But a hazy vitreous humour interferes with an oph. thalmoscopic examination for cataract. I am not able to say what are the distinctive marks or signs between slight general haze of the lens and that of the humour; and this is no more than we should suspect, when we call to mind that the two bodies are in actual contact, differ little in density, and that the one is placed immediately behind the other. I have more than once thought that $I$ had succeeded in discovering a means of distinction, but more experience disappointed my expectations. In any instance, therefore, when the presence of a diseased vitreous humour is suspected, the pupil should be examined under concentrated light, obliquely and directly, and if opacity be not detected in the lenswhich most assuredly it will be, if this part be so changed as to produce any decided loss of sight, or any ophthalmoscopic effect-to alteration in the vitreous humour must the morbid change be attributed.

\section{CASE OF BRONZED SKIN SUCCESSFULLY TREATED.}

By Thomas Taylor, Esq., Cricklade.

ON the 27th of June, 1861, I was called upon to visit Isaac Matthews, a carpenter by trade, stoutly made, of sanguine temperament, and about 36 years of age. I found he had been unwell for some time, and had been obliged to leave his work the last fortnight, simply from weakness. He was unable to walk across the room without assistance; he was in no pain, but felt an uneasiness about the loins; his appetite was not good ; the bowels were regular, and the secretions from the bowels and kidneys healtby. The skin of the face and hands was highly bronzed, and had become so about the time he left his work: he said his friends had remarked of what a curious colour he was. He was advised to keep perfectly quiet, and do nothing whatever to produce fatigue, by attempting to walk or otherwise ; to take sugar in large quantities with everything he drank; and to take five grains of iodide of potassium three times a day, in water.

July 1st. He was much the same. He did not find the medicine to disagree in any way, and was ordered to continue the same.

July 5th. He felt better before he was up this morning, and thought he should be able to walk; but on getting up felt as weak as ever. He was ordered to continue the iodide of potassium three times a day in four ounces of compound decoction of sarsaparilla.

July 12 th. He was rather stronger, and walked rather better: the complexion was rather improved. He continued to mend under this treatment until the $22 \mathrm{nd}$ when he had a severe rigor, which returned the next and following day, succeeded by fever, which reduced his strength.

On the 24th he had five grains of disulphate of quina twice a day, with croton oil liniment to the loins.

On the 7th of August his febrile state had left him, but was succeeded by a bronchial cough which was relieved in a few days; so that on the 9th he began taking the syrup of iodide of iron in half-drachm doses 
three times a day, continuing to take sugar in large quantities. Under this treatment he went on improving daily; his complexion became of its natural colour; his strength increased, so that he was enabled to walk a mile with the assistance of a walking stick, by the 19th; and by the end of the month, he walked to Cricklade, a distance of nearly five miles.

On the 9th of September he again walked to Cricklade, and appeared quite to have recovered his health and strength, and the next day resumed his work.

I heard nothing of him again, until the 5th of Febru. ary last, when I was requested to visit him, as it was thought he had a return of his complaint. On seeing him, however, I was happy to find that he was sutfering from an attack of rheumatism, having got wet away from home three times the week before, and being unable to change his clothes. This yielded very readily to treat ment in a few days, when he resumed his work, and has continued well to the present time.

\section{ON SOME AFFECTIONS OF VISION APPA RENTLY OF SYPHILITIC ORIGIN : WITH THE OPHTHALMOSCOPIC APPEARANCES.}

By R. Hibbert Taylor, M.D., Senior-Surgeon to th Liverpool Eye and Ear Infirmary, and Lecturer on Ophthalmic Medicine and Surgery to the Royal Infirmary Medical School.

ANy one who has seen much of diseases of the eye, especially as they present themselves in public institu tions for that class of maladies, must have had his attention drawn occasionally to cases apparently of syphilitic origin, in which the failure of vision could not be accounted for by any appreciable change in the external structures of the eyeball. There is probably no redness of the superficial tissues present, nor any trace of iritis, as usually manifested by thickening and loss of brilliancy of the iris, and adhesion of its pupillary border to the capsule of the lens.

On inquiring into the history of these cases, it will be found that syphilitic affections, both primary and secondary, have been observed, and generally at periods rather remote from the occurrence of the symptoms now com plained of. Among the secondary symptoms previously noted, iritis may or may not have occurred; and if it has been present, generally all traces of its existence have disappeared.

My attention was first awakened with regard to such cases many years ago, and I confess that for a lon period they seemed very puzzling. We, and I speak of those of my own starding in age, have been so accustomed, from the influence of early professional tuition, and the habits of observation founded upon it to regard syphilitic affections of the eye, as limited almost exclusively to one disease, iritis, that it was with difficulty one could shake off this inherited belief, and rise to the wider and, I believe, juster view of the subject, which these cases suggest. For myself, I must admit that it was only step by step that I was irresistibly led to the conclusion that the influence of the syphilitic poison upon the tissues of the eye possessed a more extended range than had hitherto been assigned to it, and that the deeper tissues of the choroid, retina, and vitreous humour, were probably liable to have their structures deranged and their functions impaired in like manner with those more open to inspection.

The use of the ophthalmoscope, which has already rendered such invaluable service in investigating the pathology of the deep structures of the eyeball, has elearly shown that these surmises were well founded, and that the failure of vision in these instances was amply accounted for by the structural changes whieh we have thus been enabled to detect.
In a recent number of the Medical Times and Gazette, seven cases are narrated, occurring in the practice of the Royal London Ophthalmic Hospital, which illustrate this subject on various points, and are worthy of the atten. tion of those who feel an interest in it. In one case, there was dimness of vision of one eye, following primary and secondary syphilitic symptoms which had occurred some months previously, but had then nearly disappeared, and there was no iritis. Examination with the ophthalmoscope revealed a congested and hazy condition of the retina, with the appearance of a thin gauze before it. The patient was treated with mercurials; till the mouth was slightly affected, and afterwards a milder action was kept up with some intermissions during several months. At the expiration of this period, the sight was nearly perfectly restored in the affected eye, and the morbid ophthalmoscopic appearances bad vanished. In another instance the symptoms in the eye first showed themselves six months after the primary infection; and at the period of admission to the hospital, a well-marked syphilitic rash existed upon the shoulders. Both eyes were equally affected in this case, and the symptoms complained of were $\operatorname{dim}$ muscæ, and the ap. pearance of clouds of smoke. The patient was unable to read the largest type, or tell the hour by the clock. The attack commenced rather suddenly, first in one eye, and shortly afterwards in the other. The ophthalmoscope showed a turbid vitreous humour in each eye, with numerous white silvery films floating in its structure. The patient, a married woman, was nursing an infant; but except being somewhat reduced by lactation, was in fair health. Mercury was prescribed, and the baby to be weaned; but unfortunately no record of the result is given.

In another case, the minute details of which I need not repeat, the affection of the eyes followed the primary syphilitic disease, after an interval of several years, and examination with the ophthalmoscope disclosed exten. sive structural changes in the choroid, optic disc, and retinal vessels; so great as to preclude any hope of the restoration of sight.

I need not further multiply examples from this source, as they all tend, more or less, to show the occurrence of serious structural changes in the deep tissues of the eye materially affecting vision, and taking place at periods more or less remote from the infection of primary syphilis. The point of most interest, regarding such cases, is this, that, in general, they do not present any obvious external appearance of their syphilitic origin, and unless the history of each has been minutely inquired into, its real nature would be misunderstood, and the treatment most likely to be effectual would not be adopted. Nor without the aid of the ophthalmoscope, could we detect those changes in the deep tissues which give no outward visible sign of their existence, and which yet are sometimes so extensive and important, as to be wholly irreparable.

The following case, which I recently met with in private, illustrates several of the points which appear to be characteristic of this form of secondary disease, and affords, in addition, a good example of the value of the ophthalmoscope, as without its aid it would have been impossible to refer the failure of vision to its true cause, in partial disorganisation of the deep tissues of the eyeball.

Mr. W., a tall, stout, soldier-looking man, connected with the land-transport corps in India, and twenty-two years resident in that country, consulted me about a month ago, complaining of weakness and wateriness of both eyes, with defective vision, especially in the right. He stated that about five years ago, while in a hor district of India, he first observed an appearance of a glare, like stars, before the right eye; and oljects looked at seemed to be more distant than they really were. Vision with this eye was also indistinct; but he was 